\author{
by Christian Enss \\ Coordinator of the European Microkelvin Platform - DOI: https://doi.org/10.1051/epn/2021301
}

\title{
The European Microkelvin Platform
}

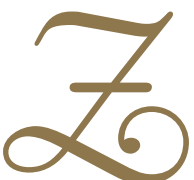

eroing in on zero to create new research opportunities in fundamental physics and materials science and to advance and exploit novel technologies are the aims of the European Microkelvin Platform (EMP). This is a consortium of eight leading ultralow-temperature laboratories and nine technology partners and companies across Europe.

The environment of ultralow temperatures, where the thermal energy is so small that the quantum nature of materials becomes evident on large scales, is and has been a breeding ground of innovations. A well-known example is superconductivity - a state of matter, first observed at very low temperatures in elementary metals, is now a vast field of research of large societal importance, as evidenced by its use in power generation, commodity searches, transportation, and medical imaging, to name just a few. Typical for the quantum technology field, this list grows constantly, as methods based on superconductivity advance and new materials are discovered. Superconductivity is just one example of many extraordinary states of matter that can ideally be explored or even discovered at ultra-low temperatures, and which have the potential to influence everyday life by leading to novel technologies that are hard to envision today. This is very much a frontier field and as the quest for even lower temperatures continually advances, as yet unknown, novel and potentially exploitable states of matter will inevitably emerge.

The EMP unites, coordinates and reinforces all existing European large-scale ultralow temperature research infrastructures in a single body and make them available to the scientific community. The individual members offer a diverse and comprehensive portfolio of experimental expertise, with eight of them opening their facilities to external researchers across the disciplines from academic institutions and companies who do not have their own infrastructure. Combined, the EMP laboratories offer the most comprehensive portfolio of cryogenic facilities worldwide. Users can apply for measuring time at EMP to pursue their own scientific ideas and projects. User access is supported by the European Union's Horizon 2020 Research and Innovation Programme. Approved user projects at EMP facilities are free of charge. In addition, travel as well as local expenses of users are covered and the EMP provides logistical and technical support if needed (see: http://www.emplatform.eu/).

A very important component of the EMP program comprises innovation projects, which are carried out in partnership between academic institutions and companies. These have been initiated to maximize the impact of the EMP research and to expedite bringing to market new products based on the technology arising from the program as quickly as possible. Several new devices based on quantum technology have been developed, enabling ultra-sensitive energy detection, magnetic flux measurement, and amplification at the quantum limit to name just a few. The consortium has already launched its first new spin-off company, "Basel Precision Instruments" from Basel University. The initial success of several of their new instruments exceeded all expectations, with the first production series being briskly sold out, necessitating a rapid ramp-up in production.

The overall objectives of the consortium are to enhance and widen the integration of the leading ultralow temperature facilities in Europe under the umbrella of the European Microkelvin Platform which will lead to strengthening Europe's international leadership in ultralow temperature studies and technology.

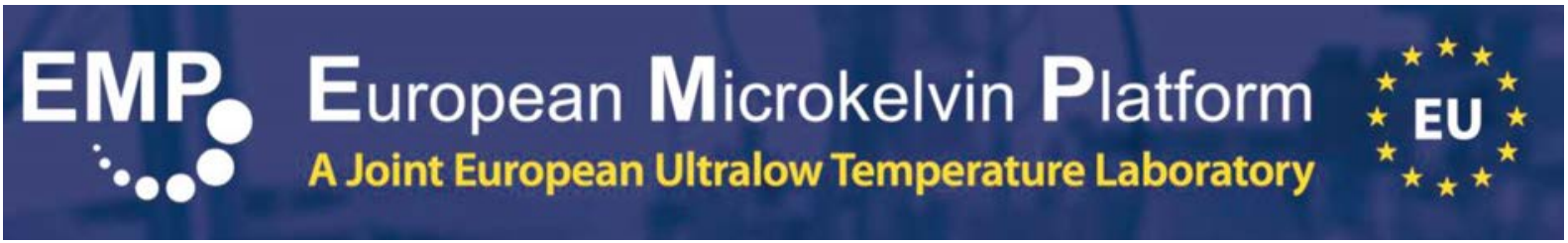

